\title{
Enhancing Low-Wavenumber Information in Reflection Waveform Inversion by the Energy Norm Born Scattering
}

\author{
Guanchao Wang, Tariq Alkhalifah, Shangxu Wang
}

\begin{abstract}
Full waveform inversion (FWI) plays a central role in the field of exploration geophysics due to its potential in recovering the properties of the subsurface at a high resolution. A starting model with ample long-wavelength components is essential for the success of most FWI algorithms. Reflection waveform inversion (RWI) is one popular way to invert for the long-wavelength velocity components from the short offset seismic data by decomposing the gradient of FWI into migration and tomographic terms. However, the transmitted part of Born scattering in conventional RWI still produces high-wavenumber artifacts, which would hinder its convergence. Thus, in this letter, an efficient non-transmission energy norm Born scattering is used in RWI to overcome the drawbacks of conventional RWI. Finally, we use numerical examples to show that the energy norm Born scattering can provide clean reflection energy from the reflector and enhance the low-wavenumber information in the RWI gradient.
\end{abstract}

Index Terms-Full waveform inversion, reflection waveform inversion, energy norm Born scattering, low-wavenumber information, reflection wavepath.

\section{INTRODUCTION}

$\mathrm{F}$ ull waveform inversion (FWI) was initially suggested to mainly invert for a high-resolution velocity structure due to its ability to perform the inversion process on all the waveform information, including diving waves, primary reflection waves and multi-scattering, simultaneously. After decades of development, the FWI theory has been widely migrated to recover density, impedance, and anisotropy parameters [1]-[4]; or map the permittivity and conductivity distributions in the Earth's interior [5]-[7]. However, as a strongly nonlinear inverse problem, FWI usually suffers from the cycle-skipping problem when initial models are crude and low-frequency components of the data are unavailable [8]-[11].

It has been well-known that both low-wavenumber (reflection and refraction/transmission kernels) and highwavenumber (migration kernel) components reside in the

We greatly appreciate the financial support of the National Key Research Development Program of China (2018YFA0702500-2018YFA0702504). Guanchao Wang wishes to thank the China Scholarship Council for support to study abroad and the SWAG in KAUST for the collaborative environment.

Guanchao Wang is with China Railway Design Corporation, National Engineering Laboratory for Digital Construction and Evaluation Technology of Urban Rail Transit, Tianjin 300251, China (Email: wgcupc@163.com.). gradient of conventional FWI [12], [13]. The transmission energy contains sufficient low-wavenumber information and can well illuminate a target region, but it only contributes to the recovery of the shallow part unless the seismic data includes quite long offsets with rich refraction and diving waves. The migration kernel of reflection, on the other hand, can update the deep part of the model, but it mainly holds high-wavenumber components. Conversely, the reflection kernel inverts for the low-wavenumber of deep part; however, it has generally weak energy compared to the high-wavenumber migration term, which causes the conventional FWI process to fall into a local minimum.

A slew of strategies has been developed to promote the lowwavenumber components update for the deep part. Migrationbased traveltime tomography [14], [15] or migration velocity analysis [16], [17] were first suggested to build a background model that provides the correct kinematic information and is required by the regular gradient-based FWI. A more advanced velocity model building method is based on reproducing the reflections using the migration/de-migration process, which allows us to update the wavepath, and referred to as reflection waveform inversion (RWI) by Xu et al. [18]. To avoid the need for a least-squares reverse time migration in RWI, several studies adopt the cross-correlation based objective function into RWI [19], [20]. Further using the RWI machinery, Alkhalifah and $\mathrm{Wu}$ [21] obtained additional low-wavenumber components from the multi-scattering energy wavepath in the frequencydomain. In addition, reflection FWI was extended by Wang et al., [22], [23], Guo and Alkhalifah [24], Li et al., [25] to elastic media. The aforementioned RWI strategy can improve the reflection kernel, however, the gradient of RWI often still includes high-wavenumbers generated from the crosscorrelation of the down-going background wavefield and the transmission part of Born scattered wavefield. To attenuate the unwanted high-wavenumber artifacts, we can separate up- and down-going wavefields in the Born scattering [26] or precondition the gradient by using a scattering angle-based filter [27]. These approaches, however, impose additional cost to the inversion.

Tariq Alkhalifah is with King Abdullah University of Science and Technology (KAUST), Thuwal 23955-6900, Jeddah, Kingdom of Saudi Arabia (Email: tariq.alkhalifah@kaust.edu.sa.).

Shangxu Wang is with China University of Petroleum-Beijing (CUPB), State Key Laboratory of Petroleum Resources and Prospecting, CNPC Key Laboratory of Geophysical Exploration, Changping 102249, Beijing, China (Email: wangsx@cup.edu.cn.). 
In this paper, we use the energy norm Born scattering [28] in the frequency-domain RWI instead of the conventional Born scattering. The energy norm Born scattering can easily isolate the scattered component from the Born wavefield and produce a gradient free of high-wavenumber artifacts. We further update both the background velocity and the short wavelength velocity perturbation simultaneously, and use the inverted velocity perturbation as the source of scattering in the RWI process as opposed to a full frequency band least-squares migration image, which requires a lot of computation.

In Section II, we give a brief introduction of the energy norm Born scattering with a single interface model example. Next, the detailed RWI workflow based on the energy norm Born scattering is described. In the end, a modified Marmousi model test demonstrates the effectiveness of the proposed algorithm.

\section{THEORY}

In this section, we will establish the concept of the energy norm Born scattering, and its main advantage over regular Born. Then, we will incorporate it into reflection waveform inversion (RWI).

\section{A. Non-transmission Energy Norm Born Scattering}

We first share a brief review of the inverse scattering imaging condition or referred to lately as well as energy norm imaging condition, which can be stated in the frequency-domain as [29], [30],

$$
I_{E}(\boldsymbol{x})=\sum_{\omega} \frac{\omega^{2}}{v(\boldsymbol{x})^{2}} u_{s}(\boldsymbol{x}) u_{r}(\boldsymbol{x})+\nabla u_{s}(\boldsymbol{x}) \cdot \nabla u_{r}(\boldsymbol{x})
$$

where $u_{s}(\boldsymbol{x})$ and $u_{r}(\boldsymbol{x})$ are the source and receiver wavefields in the frequency-domain, respectively, $v(x)$ is the velocity in the model space, $\omega$ represents the circular frequency and $\nabla$ is the space gradient operator. In the $2 \mathrm{D}$ case, $\nabla=\left(\frac{\partial}{\partial x}, \frac{\partial}{\partial z}\right)$. We can rewrite equation 1 in compact form,

$$
I_{E}(\boldsymbol{x})=\sum_{\omega} \square u_{s}(\boldsymbol{x}) \cdot \square u_{r}(\boldsymbol{x})
$$

where the energy operator $\square u_{s}(\boldsymbol{x})=\left(\frac{i \omega}{v}, \frac{\partial}{\partial x}, \frac{\partial}{\partial z}\right) u_{s}(\boldsymbol{x})$ acting on the source wavefield, and $\square u_{r}(\boldsymbol{x})=\left(\frac{-i}{v}, \frac{\partial}{\partial x}, \frac{\partial}{\partial z}\right) u_{r}(\boldsymbol{x})$ acting on the receiver wavefield. The main feature of the energy norm imaging condition, as observed in equations 1 and 2 , is that it can attenuate the backscattering artifacts corresponding to waves propagating along the same path, which provide clean reflections, automatically. For detailed development of the energy norm imaging condition, we refer the readers to Rocha et al. [30].

The adjoint of the traditional imaging condition is Born modeling; Similarly, the adjoint of the energy norm imaging condition generates the energy norm Born scattering, which yields pure reflection energy. We will demonstrate this property in the numerical example. Using the concise energy operator, we can easily express the adjoint of equations 1 and 2, regarded as energy norm Born scattering (take the source-side energy norm Born scattering for example) [30],

$$
d_{m}\left(\boldsymbol{x}_{\boldsymbol{r}}, \omega\right)=G\left(\boldsymbol{x}_{\boldsymbol{r}}, \boldsymbol{x}, \omega\right) \nabla^{T} \boxminus u_{s}(\boldsymbol{x}) I_{E}(\boldsymbol{x}),
$$

where $G\left(\boldsymbol{x}_{\boldsymbol{r}}, \boldsymbol{x}, \omega\right)$ is the Green's function from space location $\boldsymbol{x}$ to the receiver location $\boldsymbol{x}_{\boldsymbol{r}}$. The virtual source scattering term, $\square^{T} \square u_{s}(\boldsymbol{x}) I_{E}(\boldsymbol{x})$, of the de-migrated data, $d_{m}\left(\boldsymbol{x}_{\boldsymbol{r}}, \omega\right)$, can be further derived as,

$$
\begin{gathered}
\nabla^{T} \cdot u_{s}(\boldsymbol{x}) I_{E}(\boldsymbol{x})=I_{E}(\boldsymbol{x}) \frac{\omega^{2}}{v^{2}} u_{s}(\boldsymbol{x})+\nabla \cdot\left[I_{E}(\boldsymbol{x}) \nabla u_{s}(\boldsymbol{x})\right] \\
=I_{E}(\boldsymbol{x}) \frac{\omega^{2}}{v^{2}} u_{s}(\boldsymbol{x})+I_{E}(\boldsymbol{x}) \nabla \cdot \nabla u_{s}(\boldsymbol{x})+\nabla I_{E}(\boldsymbol{x}) \cdot \nabla u_{s}(\boldsymbol{x}) \\
=I_{E}(\boldsymbol{x}) \frac{\omega^{2}}{v^{2}} u_{s}(\boldsymbol{x})+I_{E}(\boldsymbol{x}) \nabla^{2} u_{s}(\boldsymbol{x})+\nabla I_{E}(\boldsymbol{x}) \cdot \nabla u_{s}(\boldsymbol{x}) \\
=\nabla I_{E}(\boldsymbol{x}) \cdot \nabla u_{s}(\boldsymbol{x})
\end{gathered}
$$

We highlight the main feature of the energy norm Born scattering with a single interface model. Fig. 1 shows the wavefields for the conventional Born scattering (Fig. 1a) and the energy norm Born scattering given in equation 3 (Fig. 1b). Fig. 1 demonstrates the key property of the energy norm Born scattering in attenuating transmission energy. To highlight the importance of this feature for RWI, we calculate the corresponding reflection kernels. Figs $2 \mathrm{a}, 2 \mathrm{~b}$ and $2 \mathrm{c}, 2 \mathrm{~d}$ show such kernels computed using the conventional and energy norm Born scattering, respectively. We see that the migration ellipse artifacts in RWI, which comes from the cross-correlation of transmission and reflection components, is well attenuated in the application of the energy norm Born scattering. The lowwavenumber reflection kernels are free of high vertical wavenumber components, which is expected to lead to faster convergence in the inversion. There is another type of energy norm Born scattering, which is associated with the nonreflection wave equation. Readers can find details of it in Sun and Alkhalifah's paper [28], in this letter we focus on the purely reflection one.

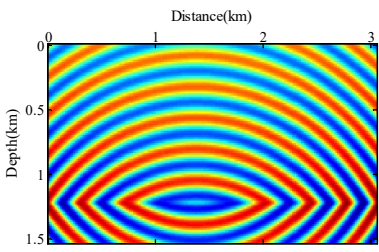

(a)

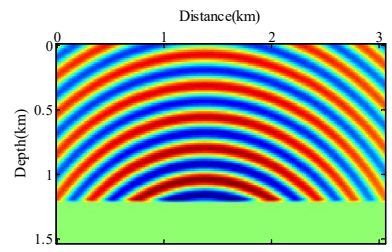

(b)
Fig. 1 The frequency-domain Born scattered wavefield for a single interface model. (a) The conventional Born scattering operator; (b)the energy norm Born scattering operator.

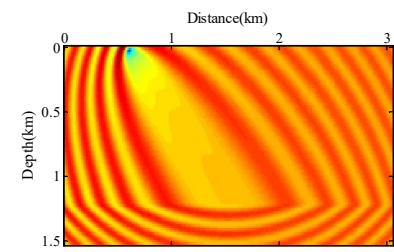

(a)

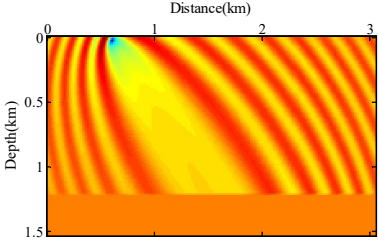

(c)

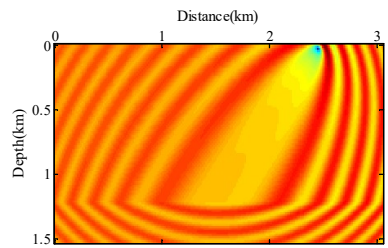

(b)

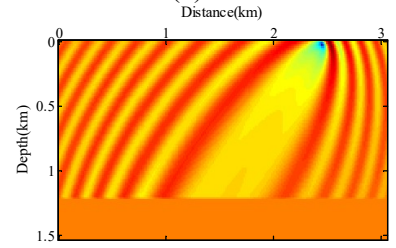

(d) 


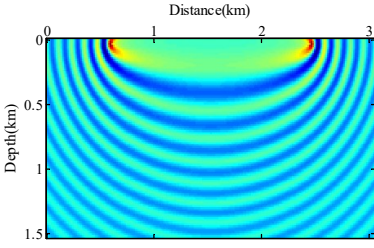

(e)

Fig. 2 The source and receiver side transmission kernels for conventional Born scattering ( $a$ and $b$, respectively), and for the energy norm Born scattering ( $c$ and d, respectively). e) the conventional FWI kernel.

\section{B. Reflection Waveform Inversion with energy norm Born scattering}

Fig. 2e shows the conventional FWI kernel, which includes the low-wavenumber wavepath from the source to the receiver. This transmission component referred to as "banana kernel," can also be seen in the RWI kernels reflecting from the interface (a combination of Figs $2 a$ and $2 b$, which has a shape of a rabbit ear). It implies that the transmission kernel in Fig. 2e can update the low-wavenumber as well but limited to a shallow area unless we have data with a large offset to depth ratio. This kind of low-wavenumber information is always neglected in conventional RWI. To utilize both low-wavenumber components (FWI and RWI), we use the following misfit function proposed by Alkhalifah and $\mathrm{Wu}$ [21],

$$
\begin{gathered}
E(v(\boldsymbol{x}), \delta v(\boldsymbol{x}))=\frac{1}{2} \sum_{s, r, \omega} \| d_{s y n}\left(\boldsymbol{x}_{\boldsymbol{r}}, \omega\right)+d_{m}\left(\boldsymbol{x}_{\boldsymbol{r}}, \omega\right)- \\
d_{o b s}\left(\boldsymbol{x}_{\boldsymbol{r}}, \omega\right) \|^{2}
\end{gathered}
$$

where $d_{o b s}\left(\boldsymbol{x}_{\boldsymbol{r}}, \omega\right)$ is the observed data, $d_{\text {syn }}\left(\boldsymbol{x}_{\boldsymbol{r}}, \omega\right)$ and $d_{m}\left(\boldsymbol{x}_{\boldsymbol{r}}, \omega\right)$ are the simulated data using the background velocity $v$ and Born simulated data using the velocity perturbation, $\delta v$, respectively. To obtain a full frequency band image $I_{E}(\boldsymbol{x})$ using frequency-domain wavefields requires substantial computational cost. Thus, in this study, the velocity perturbation $\delta v(\boldsymbol{x})$ corresponding to a single frequency (or several frequency slices) was used for the de-migration process (Equation 4).

The background and perturbed velocities are updated simultaneously by minimizing the objective function 5 . We deduce the gradient of the functional with respect to velocity perturbation using the adjoint state method [31],

$$
\nabla_{\delta v} E(\boldsymbol{x})=-\frac{2}{v^{3}} \sum_{s, r, \omega}\left[\omega^{2} u_{0}(\boldsymbol{x}, \omega) * u^{*}(\boldsymbol{x}, \omega)\right]
$$

and the gradient of the background velocity,

$$
\begin{array}{r}
\nabla_{v} E(\boldsymbol{x})=-\frac{2}{v^{3}} \sum_{s, r, \omega} \omega^{2}\left[u_{0}(\boldsymbol{x}, \omega) * u^{*}(\boldsymbol{x}, \omega)+\delta u_{0}(\boldsymbol{x}, \omega) *\right. \\
\left.u^{*}(\boldsymbol{x}, \omega)+u_{0}(\boldsymbol{x}, \omega) * \delta u^{*}(\boldsymbol{x}, \omega)\right]
\end{array}
$$

where $u_{0}(\boldsymbol{x})$ and $u^{*}(\boldsymbol{x})$ represent the forward and backward adjoint background wavefields, respectively. The $\delta u_{0}(\boldsymbol{x})$ and $\delta u^{*}(\boldsymbol{x})$ are the receiver- and source-side energy norm Born scattering wavefields using $\square^{T} \square u_{0}(\boldsymbol{x}) \delta v(\boldsymbol{x})$ and $\square^{T} \square$ $u^{*}(\boldsymbol{x}) \delta v(\boldsymbol{x})$ as the source term, respectively (Equation 3 ).

An accurate velocity perturbation image is needed for a stable update of the background velocity. Therefore, we optimize the $\delta v(\boldsymbol{x})$ for each frequency by fitting it to the residuals. Since the background model is stationary, such optimizations in the frequency-domain are cheap, requiring one Stiffness matrix inversion. The background part $v(\boldsymbol{x})$ is updated using the RWI kernel. The detailed workflow of our suggested energy norm Born scattering RWI is given in Table 1 .

Tabe1. RWI workflow with energy norm Born scattering

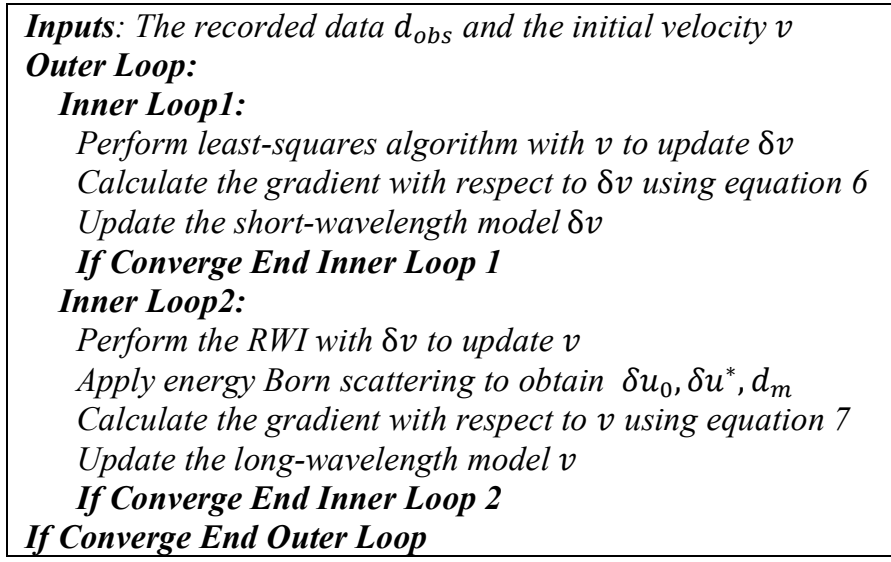

\section{EXAMPLE}

We evaluate the effectiveness of the proposed energy norm Born scattering RWI method on the modified Marmousi model, which is shown in Fig.3. The Marmousi model here has a size of $n x * n z=465 * 148$ with a grid interval of $20 \mathrm{~m}$ in both the horizontal and vertical directions, respectively. 45 shots are positioned on the surface at a $200 \mathrm{~m}$ interval. The wavefields are recorded by 465 receivers covering the entire model at the surface.

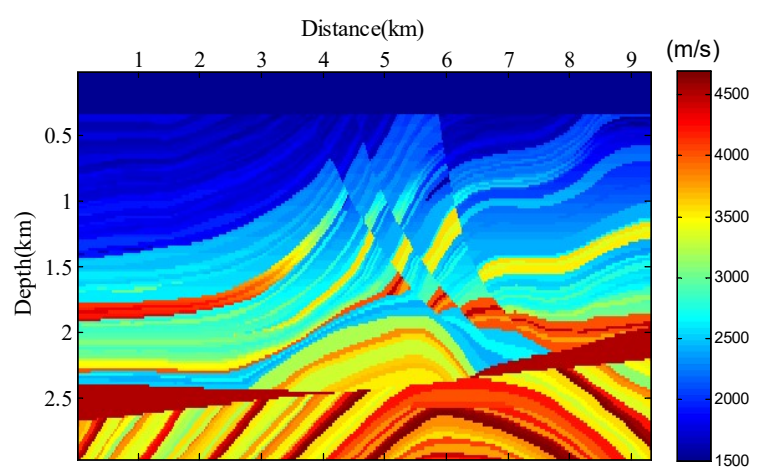

Fig. 3 The true modified Marmousi model.

Three frequencies $(3 \mathrm{~Hz} 3.5 \mathrm{~Hz}$ and $4 \mathrm{~Hz}$ ) were used in this experiment to demonstrate its effectiveness with limited data. The initial model shown in Fig. 4a for this numerical experiment is a linearly increasing velocity, $1.5 \mathrm{~km} / \mathrm{s}$ at the top and $4.0 \mathrm{~km} / \mathrm{s}$ at the bottom. We first optimize the velocity perturbation by migrating the data $\left(d_{\text {syn }}\left(\boldsymbol{x}_{\boldsymbol{r}}, \omega\right)-\right.$ $\left.d_{o b s}\left(\boldsymbol{x}_{\boldsymbol{r}}, \omega\right)\right)$ under the frame of a least-squares reverse migration using the initial velocity. However, with an inaccurate background velocity model $v$, the migrated perturbation image shown in Fig. $4 \mathrm{~b}$ is generally inaccurate. 
The inverted background velocity with the energy norm Born scattering is shown in Fig.5a, and the optimal velocity perturbation is shown in Fig. 5b. Obviously, the image in Fig. 5 b is considerably better than Fig. $4 \mathrm{~b}$ in positioning the perturbations. For comparison, Fig. 7a displays the conventional Born scattering RWI result starting from the linearly increasing initial model. The long-wavelength information was somewhat updated in Fig. 7a, but the highwavenumber components (black arrow) were also inverted despite the smoothing filter for the RWI gradient.
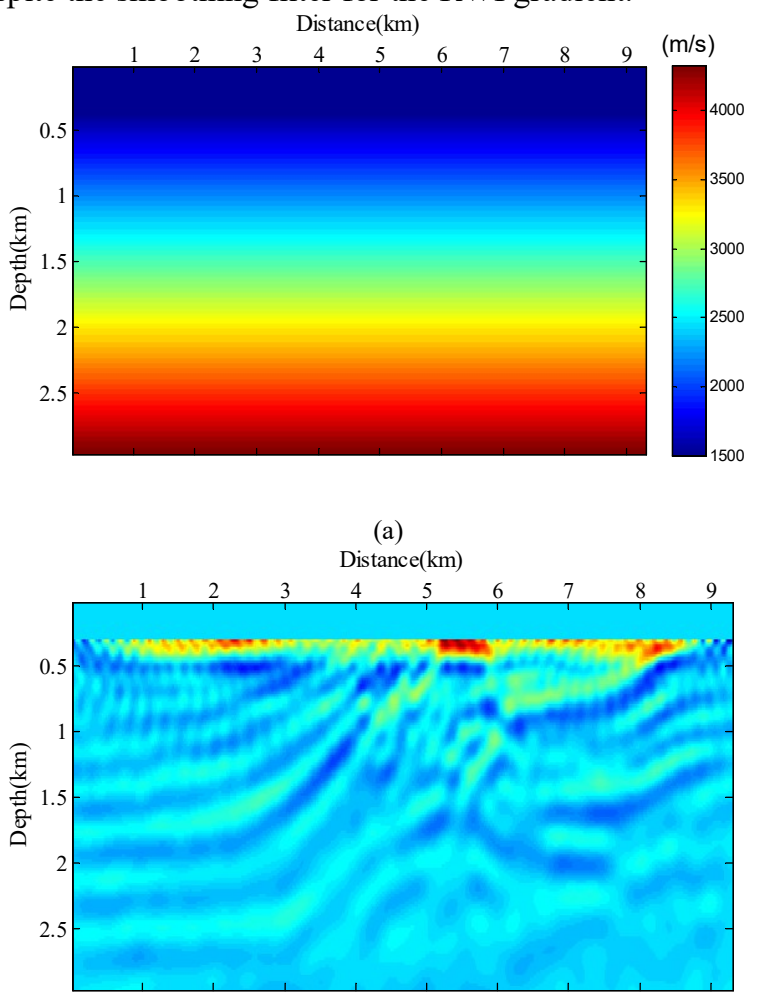

(b)

Fig. 4 (a) The linearly increasing starting model, (b) The inverted model perturbation $\delta v$ for this initial model.

To verify that the long-wavelength velocity was properly inverted by our proposed RWI, we follow the RWI with a conventional FWI using the RWI results as initial velocities. Fig. 6 is the final FWI result using Fig. 5a as the starting velocity. Since the starting model free of high-wavenumber artifacts, the inverted velocity is very close to the actual model. Fig. $7 b$ is the FWI result from Fig. 7a. In Fig. 7b, some velocity structures are mislocated, and the velocity value is not correctly inverted as well due to artifacts in the initial model (Fig.7a). As we can conclude from these results, our energy norm Born scattering RWI approach can provide a better starting model for conventional FWI to avoid cycle skipping even in the absence of the low frequencies, specifically below $3 \mathrm{~Hz}$.
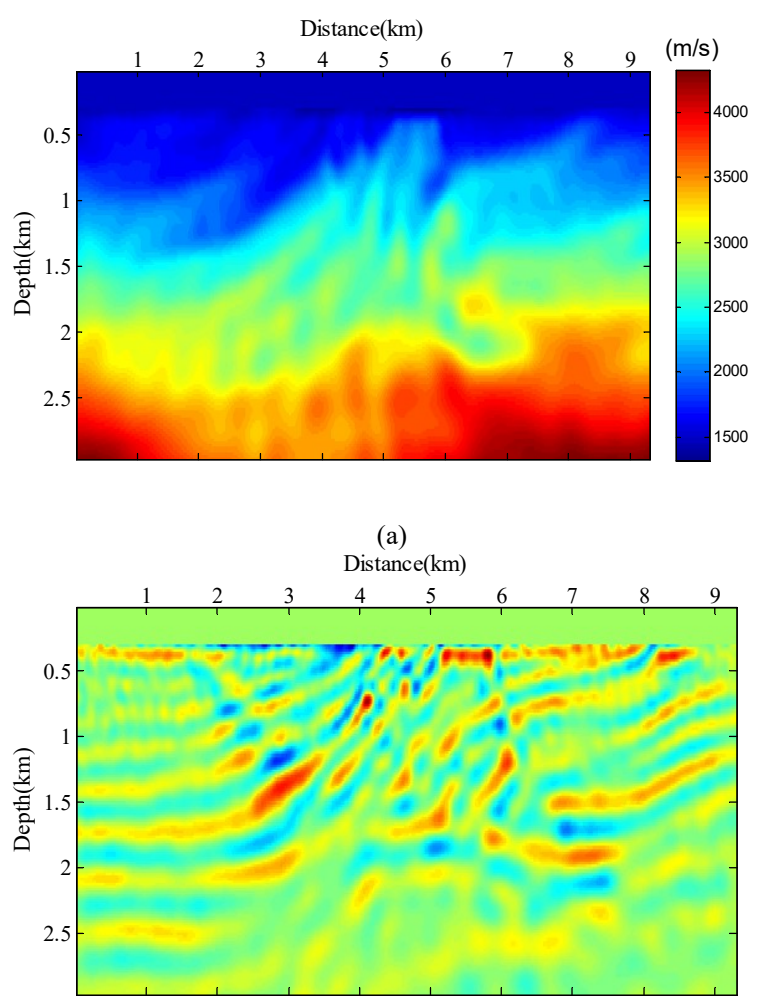

(b)

Fig. 5 (a) The inverted background model $v$ using the energy norm Born scattering based RWI, (b) the inverted model perturbation $\delta v$ for the (a).

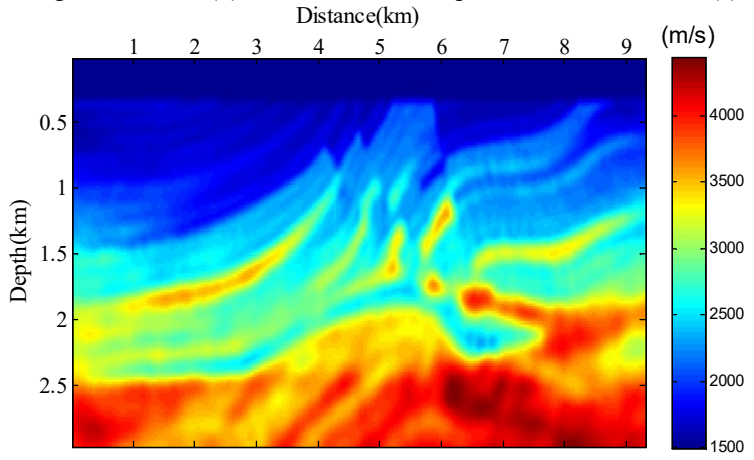

Fig. 6 The FWI result from the background model (Fig. 5a) with the inverted perturbation (Fig. 5b) added to it.

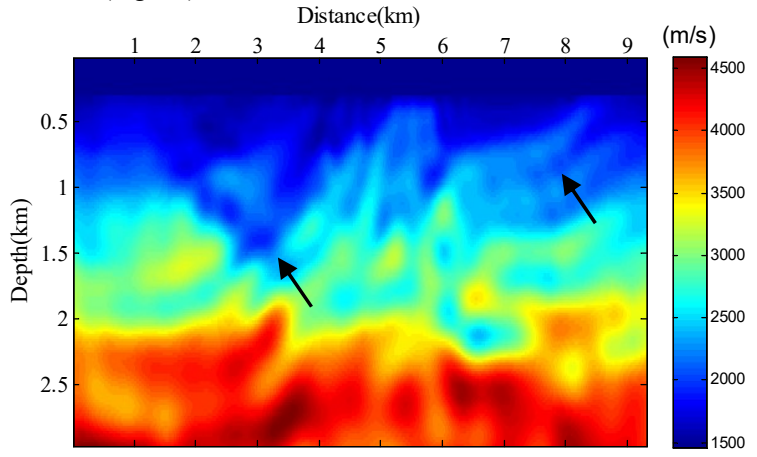

(a) 


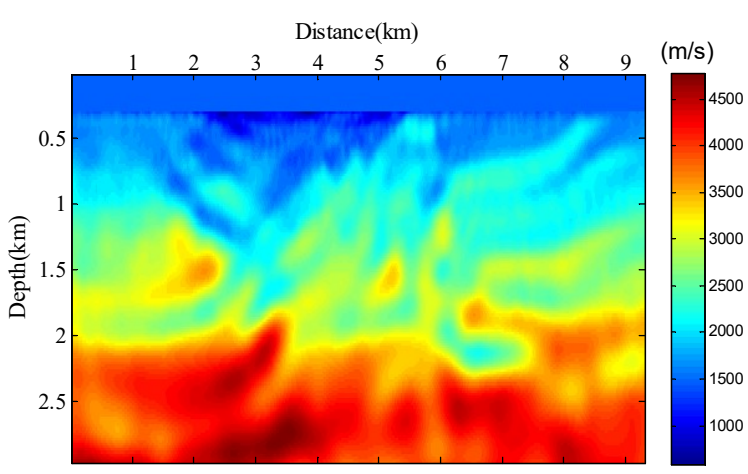

(b)

Fig. 7 (a) The inverted background model $v$ using the conventional Born scattering based RWI, (b) the final FWI result from the background model in (a).

\section{CONCLUSION}

In this letter, we developed a frequency-domain energy norm Born scattering reflection waveform inversion (RWI) algorithm to remove the high-wavenumber artifacts in the conventional RWI gradient and enhance the long-wavelength velocity update. The energy norm Born scattering formula can attenuate the transmission and produce pure reflection wavefields to compute the gradient of RWI. The inclusion of the energy Born scattering helps mitigate the high-wavenumber artifacts from our RWI results. A numerical test on the Marmousi model demonstrates that our proposed RWI can better invert the longwavelength velocity, which enables the conventional FWI to converge to a more accurate final result.

\section{REFERENCES}

[1] A. Kadu, T. Leeuwen, and W. A. Mulder, "Salt reconstruction in fullwaveform inversion with a parametric level-set method," IEEE Trans. Comput. Imag., vol. 3, no. 2, pp. 305-315, 2017.

[2] D. Dagnino, V. Sallarès and C. R. Ranero, "Waveform-Preserving Processing Flow of Multichannel Seismic Reflection Data for AdjointState Full-Waveform Inversion of Ocean Thermohaline Structure," IEEE Trans. Geosci. Remote Sens., vol. 56, no. 3, pp. 1615-1625, 2018.

[3] S. Yuan, Y. Liu, Z. Zhang, C. Luo, and S. Wang, "Prestack stochastic frequency-dependent velocity inversion with rock-physics constraints and statistical associated hydrocarbon attributes," IEEE Geosci. Remote Sens. Lett., vol. 16, no. 1, pp. 140-144, Jan. 2019.

[4] S. Yuan, S. Wang, Y. Luo, W. Wei, and G. Wang, "Impedance inversion by using the low-frequency full-waveform inversion result as a priori model," Geophysics, vol. 84, no. 2, pp. R149-R164, 2019.

[5] J. R. Ernst, H. Maurer, A. G. Green and K. Holliger, "Full-waveform inversion of crosshole radar data based on 2-D finite-difference timedomain solutions of Maxwell's equations," IEEE Trans. Geosci. Remote Sens., vol. 45, no. 9, pp. 2807-2828, 2007.

[6] G. A. Meles, S. A. Greenhalgh, A. G. Green, H. Maurer, and J. V. Kruk, "GPR full-waveform sensitivity and resolution analysis using an FDTD adjoint method," IEEE Trans. Geosci. Remote Sens., vol. 50, no. 5, pp. 1881-1896, 2012.

[7] T. Lan, H. Liu, N. Liu, J. Li, F. Han and Q. H. Liu, "Joint Inversion of Electromagnetic and Seismic Data Based on Structural Constraints Using Variational Born Iteration Method," IEEE Trans. Geosci. Remote Sens. vol. 56 , no. 1 , pp. 436-445, 2018

[8] W. Hu, J. Chen, J. Liu, and A. Abubakar, "Retrieving low wavenumber information in FWI: An overview of the cycle-skipping phenomenon and solutions," IEEE Signal Process. Mag., vol. 35, no. 2, pp. 132-141, Mar. 2018 .
[9] C. E. J. Tejero, V. Sallares, and C. R. Ranero, "Appraisal of Instantaneous Phase-Based Functions in Adjoint Waveform Inversion," IEEE Trans. Geosci. Remote Sens., vol. 1, pp. 1-13, 2018.

[10] G. Wang, S. Yuan, and S. Wang, "Retrieving Low-Wavenumber Information in FWI: An Efficient Solution for Cycle Skipping," IEEE Geosci. Remote Sens. Lett., vol. 16, no. 7, pp.1125-1129, 2019.

[11] C. Song, and T. Alkhalifah, " Efficient Wavefield Inversion With Outer Iterations and Total Variation Constraint," IEEE Trans. Geosci. Remote Sens., doi: 10.1109/TGRS.2020.2971697.

[12] A. Tarantola, "Inversion of seismic reflection data in the acoustic approximation," Geophysics, vol. 49, pp. 1259-1266, 1984.

[13] J. F. Claerbout, "Imaging the earth's interior," Oxford: Blackwell scientific publications., 1985.

[14] G. Chavent, "Data Space Reflectivity and the Migration based Travel Time approach to FWI," 79th EAGE Conference and Exhibition Workshops., 2017.

[15] Y. Yu, J. Cheng, T. Yang, S. Ruan, "Angle-Domain Differential Reflection Traveltime Tomography," IEEE Geosci. Remote Sens. Lett., to be published, 2019.

[16] W. W. Symes, and M. Kern, "Inversion of reflection seismograms by differential semblance analysis: Algorithm structure and synthetic examples," Geophys. Prospecting., vol. 42, pp. 565-614, 1994.

[17] B. Biondi, and A. Almomin, "Simultaneous inversion of full data bandwidth by tomographic full-waveform inversion," Geophysics., vol. 79, no. 3, pp. WA129-WA140, 2014.

[18] S. Xu, D. Wang, Y. Chen, Y. Zhang, and G. Lambare, "Full waveform inversion for reflected seismic data," 74th EAGE Conference and Exhibition., 2012.

[19] B. Chi, L. Dong, and Y. Liu, "Correlation-based reflection full-waveform inversion," Geophysics, vol. 80, no. 4, pp. R189-R202, 2015.

[20] Y. Ma, D. Hale, "Wave-equation reflection traveltime inversion with dynamic warping and full-waveform inversion," Geophysics, vol. 78, no. 6, pp. R223-R233, 2013.

[21] T. Alkhalifah, and Z. Wu, "Multiscattering inversion for low-model wavenumbers," Geophysics, vol. 81, no. 6, pp. R417-R428, 2016.

[22] G. Wang, S. Wang, Q. Du, and S. Yuan, "Traveltime-based reflection fullwaveform inversion for elastic medium," J. Appl. Geophys., vol. 141, pp. 68-76, 2017.

[23] G. Wang, S. Wang, J. Song, C. Dong, and M. Zhang, "Elastic reflection traveltime inversion with decoupled wave equation," Geophysics, vol. 83, no. 5, pp. R463-R474, 2018.

[24] Q. Guo, and T. Alkhalifah, "Elastic reflection-based waveform inversion with a nonlinear approach," Geophysics., vol. 82, no. 6, R309-R321, 2017.

[25] Y. Li, Q. Guo, Z. Li, and T. Alkhalifah, "Elastic reflection waveform inversion with variable density," Geophysics., vol. 84, R553-R567, 2019.

[26] F. Liu, G. Zhang, S. Morton, and J. Leveille, "An effective imaging condition for reverse-time migration using wavefield decomposition," Geophysics, vol. 76, pp. S29-S39, 2011.

[27] T. Alkhalifah, "Scattering-angle based filtering of the waveform inversion gradients," Geophys. J. Int., vol. 200, pp. 363-373, 2015.

[28] B. Sun, and T. Alkhalifah, "Wavefield separation by energy norm Born scattering," 87th Annual International Conference and Exhibition, SEG, Expanded Abstracts, pp. 1481-1485, 2017.

[29] N. D. Whitmore, and S. Crawley, "Applications of RTM inverse scattering imaging conditions," 82nd Annual International Meeting, SEG, Expanded Abstracts, pp. 1-6, 2012.

[30] D. Rocha, N. Tanushev, and P. Sava, "Acoustic wavefield imaging using the energy norm," Geophysics, vol. 81, no. 4, pp. S151-S163, 2016.

[31] R. Plessix, "A review of the adjoint-state method for computing the gradient of a functional with geophysical applications," Geophys. J. Int., vol. 167, pp. 495-503, 2006. 